\title{
The combination of blue dye and radioisotope versus radioisotope alone during sentinel lymph node biopsy for breast cancer: a systematic review
}

Pei-Sheng He${ }^{2}$, Feng $\mathrm{Li}^{3^{*}}$, Guan-Hua Li ${ }^{1}$, Can Guo ${ }^{1}$ and Tian-Jin Chen ${ }^{1}$

\begin{abstract}
Background: The combination of blue dye and radioisotope is most widely used to identify sentinel lymph nodes (SLNs) in patients with breast cancer. However, some individual studies suggested that dual tracers did not have an advantage over radioisotope alone in detecting SLNs. We performed a systematic review to investigate the added value of blue dye in addition to radioisotope.

Methods: We searched Pubmed and Embase. Prospective studies that compared the combination of radioisotope and blue dye with radioisotope alone were selected. The identification rate of SLNs and the false-negative rate were the main outcomes of interest. The odds ratios (ORs) and $95 \%$ confidential intervals (Cls) were calculated by using random-effects model.

Results: Twenty-four studies were included. The combination of radioisotope and blue dye showed higher identification rate than radioisotope alone $(\mathrm{OR}=2.03,95 \% \mathrm{Cl} 1.53-2.69, P<0.05)$. However, no statistically significant difference was revealed for patients after neoadjuvant chemotherapy ( $\mathrm{OR}=1.64,95 \% \mathrm{Cl} 0.82-3.27, P>0.05)$, or for studies with high proportion of patients with positive lymphoscintigraphy $(\mathrm{OR}=1.41,95 \% \mathrm{Cl} 0.83-2.39, P>0.05)$. Dual tracers did not significantly lower the false-negative rate compared with radioisotope alone $(\mathrm{OR}=0.76,95 \% \mathrm{Cl} 0.44-1.29, P>0.05)$.

Conclusions: Although the combination of blue dye and radioisotope outperformed radioisotope alone in SLN detection, the superiority for dual tracers may be limited for patients with positive lymphoscintigraphy or for those after neoadjuvant chemotherapy. Besides, the combined modality did not help lower the false-negative rate.
\end{abstract}

Keywords: Breast cancer, Sentinel lymph node, Blue dye, Radioisotope, Systematic review

\section{Background}

The most important prognostic factor for patients with early-stage breast cancer was the disease status of axillary lymph nodes [1]. Recently, sentinel lymph node biopsy (SLNB) has replaced axillary lymph node dissection (ALND) to be the standard procedure for axillary staging in patients with clinically node-negative breast cancer [2,3]. SLNs were defined as the first lymph nodes that received lymphatic drainage from the primary cancer. Since the early 1990s, blue dye and radioisotope have emerged as the most commonly used tracing agents to locate SLNs in breast

* Correspondence: fengl_sx@126.com

${ }^{3}$ Department of Urinary Surgery, Three Gorges Central Hospital, 165 Xincheng Road, Chongqing 404000, China

Full list of author information is available at the end of the article cancer $[4,5]$. In particular, the combined use of blue dye and radioisotope gained widespread popularity [6]. A previous survey of fellows of the American College of Surgeons showed that $90 \%$ used the combined modality [7].

Notably, blue dye injection carried the potential risks of skin tattooing, skin necrosis, and allergic reactions [8]. Approximately $2 \%$ of patients undergoing SLNB would experience allergic reactions to blue dye [9], with the most severe case presenting as hypotension [10]. Several authors argued that the added value of blue dye over radiotracer alone technique was only minimal or marginal [9-11]. The results from a large case series suggested that the marginal benefit for blue dye declined with increased surgical experience in radioisotope-mapping technique [12]. 
It is attractive to use radioisotope alone to avoid the blue-dye complications and lower the cost of hospital care. However, the current knowledge on the added value of blue dye is still based on weak evidence from scattered individual studies without universal consensus. A randomized controlled trial (RCT) has only recently been conducted to compare dual tracers with radioisotope alone in patients before neoadjuvant chemotherapy (NAC) with positive preoperative lymphoscintigraphy (LSG), which demonstrated no advantage for dual tracers in SLN detection [11]. The authors speculated that the blue dye should be added only for patients with negative LSG or those having received NAC. An evidence-based systematic review was warranted to identify patients who will particularly benefit from dual tracers, and to help inform SLNB decision-making. Thus, we conducted this systematic review regarding prospective studies on SLNB in breast cancer, aiming to gain a better understanding of the incremental value of blue dye in addition to radioisotope. Especially, the potential confounding clinical factors were explored.

\section{Methods}

Study selection

Electronic databases of Pubmed and Embase were systematically searched up to June 2015. The search terms used were: 'sentinel lymph node', 'breast cancer,' 'blue dye' or 'lymphazurin' or 'Isosulfan' or 'methylene blue' or 'patent blue, 'isotope' or 'radioisotope' or 'radiolabeled colloid' or 'radiocolloid' or 'radiotracer'. The search was restricted to human subjects and English language. All studies were critically appraised for inclusion eligibility. We also manually searched the reference lists of relevant studies.

\section{Inclusion criteria}

Studies were considered for inclusion if they fulfilled the following criteria: (1) reported the use of blue dye and radioisotope for SNLB in female breast cancer patients; (2) showed the comparison between the combination of blue dye and radioisotope with radioisotope alone; (3) reported outcomes of the identification rate of SLNs or the false-negative rate; (4) prospectively collected patients' data, designed as randomized controlled trial (RCT) or non-randomized prospective study (NPS); (5) enrolled at least 100 patients, with at least 20 patients available for each mapping strategy.

\section{Data extraction and quality assessment}

Data from the included studies were extracted independently by two authors (PSH and GHL). Any discrepancy was resolved by consensus or by discussion with a third author (FL). The following information was extracted: author and publication year, location, study design, sample size, age, clinical status of axillary nodes, NAC use, mapping materials, injection site, and proportion of patients with positive preoperative LSG. The identification rate of SLNs and the false-negative rate were directly extracted or indirectly calculated for each mapping strategy. The quality of studies were appraised by a revised 6-item scale which was derived from a previous 5-item scale [13]. Assuming that the success rate of SLN identification reached the level of $98 \%$ for dual mapping agents, and differed by $5 \%$ between dual and single tracing agents, accompanied by a Type I error probability for a two-sided test of $5 \%$ and statistical power of $80 \%$, the required sample size in each group was calculated to be approximately 300 [14]. Thus, we listed the sufficiency of sample size as one item on the quality scale. The quality assessment included the following elements: 1) describing patients' characteristics, 2) explaining reasons for withdrawal, 3) describing measures of outcomes, 4) incorporating measures of confounding factors, 5) describing the SLN technique (mapping material and injection site), and 6) enrolling at least 300 patients. The study with 5 points or more was regarded as high quality.

\section{Statistical analysis}

The odds ratios (ORs) and $95 \%$ confidential intervals (CIs) were used as statistical measures for dichotomous outcomes. They were calculated from the number of patients in each mapping modality. The identification rate of SLNs and the false-negative rate were considered as the main outcomes. The random-effects model was used to calculate the summary effect estimates [15]. The heterogeneity between studies was analyzed by the $\mathrm{I}^{2}$ statistics and Cochrane $\mathrm{Q}$ test, with $\mathrm{I}^{2}>50 \%$ and $P<0.05$ deemed as significant heterogeneity. The source of heterogeneity was explored by subgroup analysis, meta-regression and cumulative analysis. The following predefined covariates were considered into subgroup analyses: clinical node status (negative or positive), NAC use (before NAC or after NAC), proportion of patients with positive preoperative LSG ( $\geq 90 \%$ or $<90 \%$ ), sample size $(>300$ or $<300)$, data source (RCT or NPS), and injection site of mapping materials (superficial or deep). The superficial injection sites included periareolar, subareolar, intradermal, and subdermal; the deep injection sites included peritumoral, intratumoral, and intraparenchymal [16]. Meta-regression analysis was performed according to the sample size, publication year, and the proportion of patients with positive preoperative LSG. The cumulative analysis was conducted according to the publication year. The publication bias was examined visually by the funnel plot and statistically by the Egger's test. $P<0.05$ was considered to represent statistically significance. The statistical analyses were performed by the STATA 12.0 (StataCorp LP, College Station, Texas, USA).

Additionally, we pooled the false-negative rates and the incidence of adverse reactions caused mapping agents, 
which were processed by the software of Comprehensive Meta-Analysis statistical package (CMA Version 2.2, Biostat, Englewood, NJ), with the use of random-effects model.

\section{Results}

\section{Literature search}

A total of 309 citations were identified after the initial search, including 137 citations from Embase and 172 citations from Pubmed. Sixty-one duplicated records were excluded. Then we excluded reviews, case reports, editorials, studies with small sample sizes $(<100)$, and studies of irrelevant topics. Seventy-four studies were screened by titles and abstracts. After excluding 26 retrospective studies, the full-texts of 48 records were assessed for eligibility. Data on the combined mapping modality could not be obtained from 12 studies. Twelve studies enrolled patients of the duplicated cohorts. Finally, twenty-four studies were selected for meta-analyses [1, 3, 8, 10-12, 17-34]. The flow diagram of selection process was depicted in Fig. 1.

\section{Study characteristics and quality assessment}

A total of 15,462 patients with breast cancer undergoing SLNB were involved. The characteristics of 24 included studies were presented in Table 1 . The sample size ranged from 100 to 3402, with 15 studies of sample sizes over 300 and 9 studies of sample sizes below 300. Eight studies were conducted in the United States, 11 in Europe, and 5 in Asia. Six studies were designed as RCTs. However, only one RCT was primarily designed

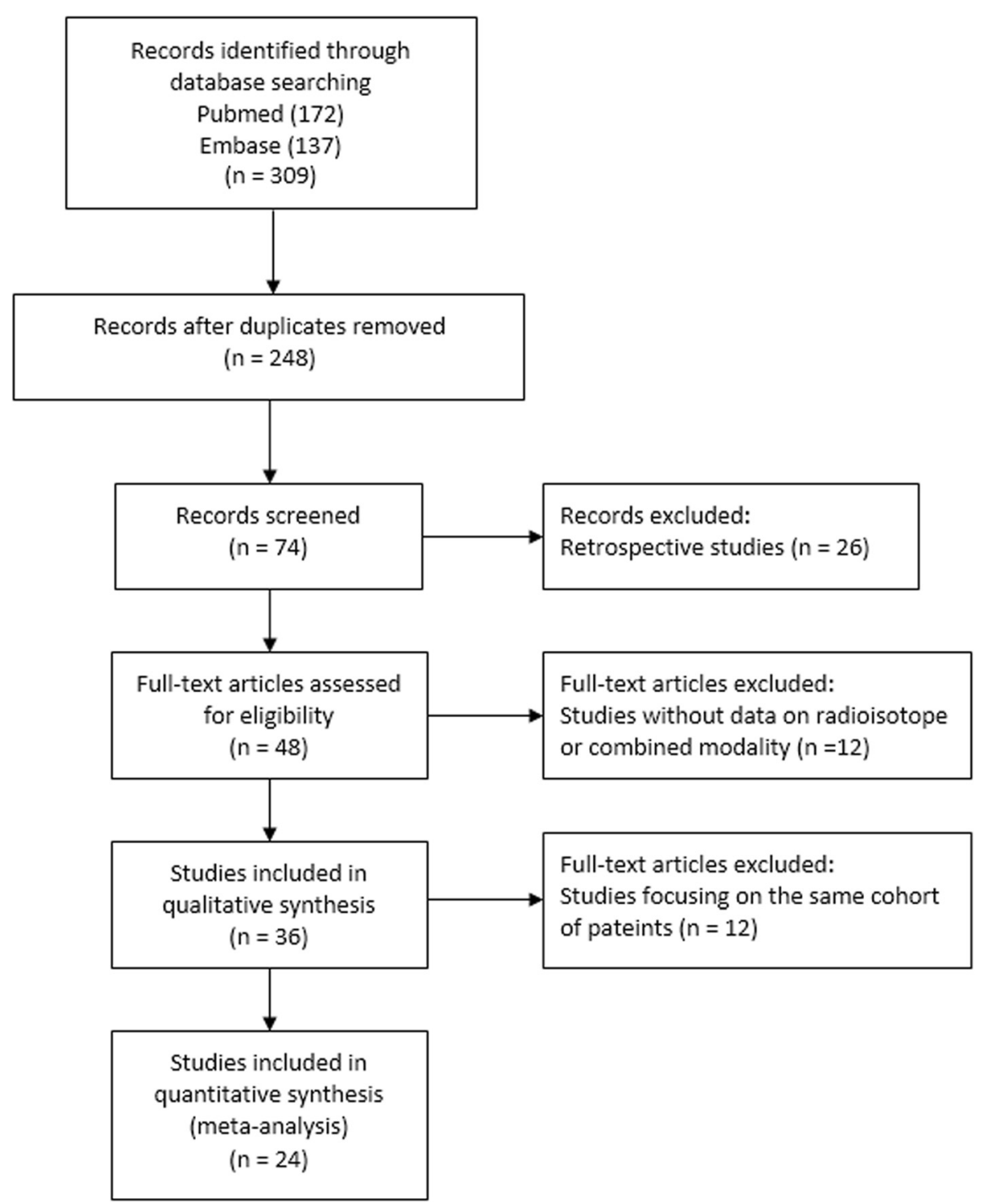

Fig. 1 The flow diagram of literature search process 
to compare radioisotope alone with the combined use of radioisotope and blue dye [11]. The comparison data were retrieved from post-hoc analyses for other five RCTs. Eighteen studies were non-randomized prospective studies. The study qualities were appraised by the revised 6item scale. The overall assessment was satisfactory with all scores ranging from 3 to 6 . Most studies clearly described the patients' characteristics (21/24), the details of SLN procedures (20/24), the confounding factors (21/24), and the measures of outcomes (24/24). However, the explanation of withdrawal or the selection process of participants were clearly stated by only few studies (7/24). The quality assessment was shown in Table 2.

\section{Identification rate of SLNs}

All of the 24 studies compared the the identification rate of SLNs between dual tracers and radioisotope alone. The pooled results demonstrated that the combined use of radioisotope and blue dye had higher identification rate of SLNs than radioisotope alone $(\mathrm{OR}=2.03,95 \% \mathrm{CI}$ 1.53-2.69, $P<0.05$ ) (Fig. 2). Significant heterogeneity was detected $\left(\mathrm{I}^{2}=64.9 \%, P<0.05\right)$. The primary subgroup analyses were conducted according to the clinical node status, NAC use (before NAC or after NAC), and proportion of patients with positive preoperative LSG ( $\geq 90 \%$ or $<90 \%$ ).

\section{The impact of clinical node status}

The clinical axillary node status was exclusively negative in 14 studies, exclusively positive in 1 study, mixed in 4 studies, and not clear in 4 studies (Table 1). In the subgroup of 14 studies with clinically node-negative breast cancer, the pooled data indicated that the use of dual tracers was superior to radioisotope alone in identifying SLNs $\left(\mathrm{OR}=2.56,95 \%\right.$ CI 1.88-3.49, $\left.P<0.05 ; \mathrm{I}^{2}=48.7 \%\right)$. However, no significant results were revealed for other subgroups (Table 3).

\section{The impact of neoadjuvant chemotherapy}

The use of NAC was clearly described by 8 studies, including 5 studies of patients before NAC, 2 studies of patients after NAC, and 2 studies with mixed populations (Table 1). Kuehn et al. reported both data for patients before NAC and those after NAC, which were extracted separately [3]. For 6 studies including patients before NAC [1, 3, 11, 21, $31,34]$, the combined use of blue dye and radioisotope showed higher identification rate than radioisotope alone (OR $=2.96,95 \%$ CI 1.78-4.94, $\left.P<0.05 ; \mathrm{I}^{2}=15.6 \%\right)$. For 3 studies including patients after NAC [3,20,27], no statistically significant difference was revealed when comparing dual tracers with radioisotope alone $(\mathrm{OR}=1.53,95 \% \mathrm{CI}$ 0.94-2.47, P > 0.05; $\mathrm{I}^{2}=31.6 \%$ ) (Table 3).

\section{The impact of preoperative LSG}

The proportion of patients with positive preoperative LSG was reported by 13 studies, ranging from 69.3 to $100 \%$ (Table 1). For 7 studies with a high proportion ( $\geq 90 \%)$ [1, $3,10,11,25,29,30]$, the pooled data revealed no statistically significant difference between dual tracers and radioisotope alone $(\mathrm{OR}=1.41,95 \% \mathrm{CI}$ 0.83-2.39, $P>0.05)$. For 6 studies with a relatively low proportion $(<90 \%)$ $[19,21-23,26,31]$, the advantage of using dual tracers was statistically significant $(\mathrm{OR}=2.99,95 \% \mathrm{CI}$ 1.99-4.48, $P>0.05$ ) (Table 3 ).

\section{Stratified analyses}

Additionally, subgroup analyses were conducted according to the data source (RCT or NPS), sample size (over 300 or below 300), location (USA, Europe, or Asia), injection site of blue dye (superficial or deep), and injection site of radioisotope (superficial or deep). Three studies used both superficial injection and deep injection [19, 22, 31]. Related data were extracted separately. The results remained significant in most subgroup analyses. However, no statistically significant difference was shown between dual tracers and radioisotope alone for patients receiving superficial injection of blue dye $(\mathrm{OR}=1.95,95 \% \mathrm{CI}$ $0.93-4.08, P>0.05)$, or for those receiving superficial injection of radioisotope $(\mathrm{OR}=2.05,95 \%$ CI 0.87-4.84, $P>0.05)$. Results for subgroup analyses were summarized in Table 3.

\section{Meta-regression and cumulative analysis}

The publication year and sample size were considered as independent variables into meta-regression analyses. No significant independent effect was detected for publication year $(P=0.37)$ or sample size $(P=0.52)$. Meta-regression was also performed for 13 studies reporting the proportion of patients with preoperative LSG, which showed a significant independent effect of this covariate $(P<0.01)$. Assumed that the surgical experience in mapping techniques increased over years, cumulative analysis was performed to investigate the effect of publication year. Notably, the advantage of combined mapping modality was stable over years (Fig. 3).

\section{Publication bias}

The funnel plot was visually symmetrical (Fig. 4). No statistical significance was detected by Egger's test $(P=0.34)$.

\section{False-negative rate}

The false-negative rate was investigated by 12 studies $[1,3,17,18,20,22-27,32]$. The pooled falsenegative rate was $7.5 \%$ (95\% CI 4.8-11.5\%), with significant heterogeneity $\left(\mathrm{I}^{2}=82.4 \%, P<0.05\right)$ (Fig. 5a). However, only 4 studies reported the comparison of false-negative rate between radioisotope alone and the 
Table 1 Characteristics of included studies

\begin{tabular}{|c|c|c|c|c|c|c|c|c|c|c|c|}
\hline Author (year) & Design & Location & $\begin{array}{l}\text { Sample } \\
\text { size }\end{array}$ & Age & $\begin{array}{l}\text { Clinical node } \\
\text { status }\end{array}$ & NAC use & Radioisotope type & Blue dye type & Site of Blue dye & Site of isotope & $\begin{array}{l}\text { Positive Preo } \\
\text { LSG, No. (\%) }\end{array}$ \\
\hline $\begin{array}{l}\text { Bass et al. (1999) } \\
{[18]}\end{array}$ & NPS & USA & 700 & 58 & Unknown & Unknown & $\begin{array}{l}\text { Filtered }{ }^{99 \mathrm{~m}} \mathrm{Tc} \text {-sulfur } \\
\text { colloid }\end{array}$ & Isosulfan & Intraparenchymal & Intraparenchymal & Unknown \\
\hline $\begin{array}{l}\text { Mariani et al. (2000) } \\
\text { [28] }\end{array}$ & NPS & Italy & 284 & 59 & Mixed & Unknown & $\begin{array}{l}{ }^{99 m} \mathrm{Tc} \text {-human } \\
\text { albumin }\end{array}$ & Patent blue & Subdermal & Subdermal & Unknown \\
\hline $\begin{array}{l}\text { Rahusen et al. } \\
\text { (2000) [30] }\end{array}$ & NPS & Netherlands & 115 & 54 & Unknown & Unknown & $\begin{array}{l}{ }^{99 m} \text { Tc-human } \\
\text { albumin }\end{array}$ & Patent blue & intradermal & Intraparenchymal & 105/115 (91 \%) \\
\hline $\begin{array}{l}\text { Derossis et al. } \\
\text { (2001) [12] }\end{array}$ & NPS & USA & 2000 & Unknown & Negative & Unknown & $\begin{array}{l}\text { Unfiltered }{ }^{99 \mathrm{~m}} \mathrm{Tc} \text { - } \\
\text { sulfur colloid }\end{array}$ & Isosulfan & Intraparenchymal & Intradermal & Unknown \\
\hline $\begin{array}{l}\text { Bauer et al. (2002) } \\
\text { [19] }\end{array}$ & NPS & USA & 332 & 55 & Negative & Unknown & $\begin{array}{l}\text { Filtered }{ }^{99 \mathrm{~m}} \mathrm{Tc} \text {-sulfur } \\
\text { colloid }\end{array}$ & Isosulfan & $\begin{array}{l}\text { Subareolar versus } \\
\text { peritumoral }\end{array}$ & Peritumoral & 195/223 (87.4 \%) \\
\hline $\begin{array}{l}\text { Ahrendt et al. } \\
\text { (2002) [17] }\end{array}$ & NPS & USA & 174 & 59 & Negative & Unknown & $\begin{array}{l}\text { Filtered }{ }^{99 \mathrm{~m}} \mathrm{Tc} \text { c-sulfur } \\
\text { colloid }\end{array}$ & Isosulfan & Intraparenchymal & Intraparenchymal & Unknown \\
\hline $\begin{array}{l}\text { Tsunoda et al. } \\
\text { (2002) [34] }\end{array}$ & NPS & Japan & 376 & Unknown & Mixed & No & $\begin{array}{l}\text { Tin colloid or } \\
\text { phytate }\end{array}$ & Unknown & $\begin{array}{l}\text { Subareolar or } \\
\text { peritumoral }\end{array}$ & Peritumoral & Unknown \\
\hline $\begin{array}{l}\text { Pelosi et al. (2003) } \\
\text { [29] }\end{array}$ & NPS & Italy & 150 & 62 & NA & Unknown & $\begin{array}{l}{ }^{99 \mathrm{~m}} \mathrm{Tc} \text {-labelled } \\
\text { Nanocoll }\end{array}$ & Isosulfan & $\begin{array}{l}\text { Periareolar or } \\
\text { subdermal }\end{array}$ & Periareolar or subdermal & 93/100 (93\%) \\
\hline $\begin{array}{l}\text { Fleming et al. } \\
\text { (2003) [22] }\end{array}$ & NPS & Ireland & 125 & $\approx 56$ & Negative & Unknown & Radiocolloid isotope & Isosulfan & Periareolar & $\begin{array}{l}\text { Intraparenchymal versus } \\
\text { intradermal }\end{array}$ & 103/125 (82.4\%) \\
\hline $\begin{array}{l}\text { Schirrmeister et al. } \\
\text { (2004) [32] }\end{array}$ & NPS & Germany & 814 & 58 & $\begin{array}{l}62.9 \% \\
\text { negative }\end{array}$ & Unknown & Radioactive colloid & $\begin{array}{l}\text { Isosulfan or } \\
\text { patent blue }\end{array}$ & Optional & Optional & Unknown \\
\hline $\begin{array}{l}\text { Lauridsen et al. } \\
\text { (2004) [24] }\end{array}$ & NPS & Denmark & 124 & 56 & Negative & Unknown & $\begin{array}{l}{ }^{99 m} \text { Tc-human } \\
\text { albumin }\end{array}$ & Patent blue & Peritumoral & Peritumoral & Unknown \\
\hline $\begin{array}{l}\text { Mamounas et al. } \\
\text { (2005) [27] }\end{array}$ & $\mathrm{RCT}$ & USA & 428 & Unknown & $\begin{array}{l}76.2 \% \\
\text { negative }\end{array}$ & Yes & Unknown & Isosulfan & Unknown & Unknown & Unknown \\
\hline $\begin{array}{l}\text { Takei et al. (2006) } \\
\text { [33] }\end{array}$ & NPS & Japan & 308 & 55 & Negative & Unknown & ${ }^{99 \mathrm{~m}}$ Tc-phytate & Patent blue & Subdermal & Subdermal & Unknown \\
\hline $\begin{array}{l}\text { Argon et al. (2006) } \\
\text { [1] }\end{array}$ & NPS & Turkey & 100 & 48 & Negative & No & ${ }^{99 m} \mathrm{Tc}$-tin colloid & Isosulfan & Intraparenchymal & Intradermal & $90 / 100(90 \%)$ \\
\hline $\begin{array}{l}\text { Low et al. (2006) } \\
\text { [26] }\end{array}$ & NPS & Australia & 113 & 56 & Negative & Unknown & ${ }^{99 m}$ Tc-sulfur colloid & Patent blue & $\begin{array}{l}\text { Intradermal or } \\
\text { subdermal }\end{array}$ & Peritumoral & 97/113 (85.8 \%) \\
\hline $\begin{array}{l}\text { Goyal et al. (2006) } \\
\text { [23] }\end{array}$ & $\mathrm{RCT}$ & UK & 842 & $18-80$ & Negative & Unknown & $\begin{array}{l}{ }^{99 m} \mathrm{Tc} \text {-albumin } \\
\text { colloid }\end{array}$ & Patent blue & Peritumoral & Peritumoral & 490/707 (69.3\%) \\
\hline $\begin{array}{l}\text { Lelievre et al. (2007) } \\
\text { [25] }\end{array}$ & NPS & France & 152 & 57 & Unknown & Unknown & ${ }^{99 m}$ Tc-sulfur colloid & Patent blue & $\begin{array}{l}\text { Subareolar or } \\
\text { peritumoral }\end{array}$ & $\begin{array}{l}\text { Intradermal and } \\
\text { intraparenchymal }\end{array}$ & 149/152 (98 \%) \\
\hline $\begin{array}{l}\text { Rodier et al. (2007) } \\
\text { [31] }\end{array}$ & $\mathrm{RCT}$ & France & 449 & $25-90$ & Negative & No & ${ }^{99 \mathrm{~m}} \mathrm{Tc}$-sulfur colloid & Patent blue & $\begin{array}{l}\text { Peritumoral versus } \\
\text { periareolar }\end{array}$ & $\begin{array}{l}\text { Peritumoral versus } \\
\text { periareolar }\end{array}$ & $353 / 432(81.7 \%)$ \\
\hline \multirow[t]{2}{*}{$\begin{array}{l}\text { Kang et al. (2010) } \\
{[10]}\end{array}$} & NPS & USA & 3402 & 56 & Negative & Mixed & ${ }^{99 m}$ Tc-sulfur colloid & Isosulfan & Unknown & Unknown & $\begin{array}{l}1566 / 1720 \\
(91.0 \%)\end{array}$ \\
\hline & NPS & USA & 696 & 57 & Unknown & Unknown & & Isosulfan & Subareolar & Subareolar & Unknown \\
\hline
\end{tabular}


Table 1 Characteristics of included studies (Continued)

\begin{tabular}{|c|c|c|c|c|c|c|c|c|c|c|c|}
\hline \multicolumn{7}{|l|}{$\begin{array}{l}\text { Johnson et al. } \\
\text { (2011) [8] }\end{array}$} & \multicolumn{5}{|l|}{$\begin{array}{l}\text { Unfiltered }{ }^{99 \mathrm{~m}} \mathrm{Tc} \text { - } \\
\text { sulfur colloid }\end{array}$} \\
\hline $\begin{array}{l}\text { Kuehn et al. (2013) } \\
\text { [3] }\end{array}$ & NPS & $\begin{array}{l}\text { Germany, } \\
\text { Austria }\end{array}$ & 1334 & 49 & Negative & Mixed & Unknown & Unknown & Optional & Optional & $\begin{array}{l}1490 / 1614 \\
(92.3 \%)\end{array}$ \\
\hline $\begin{array}{l}\text { Boughey et al. } \\
\text { (2013) [20] }\end{array}$ & $\mathrm{RCT}$ & USA & 689 & $\begin{array}{l}49(23- \\
93)\end{array}$ & Positive & Yes & Unknown & $\begin{array}{l}\text { Isosulfan or } \\
\text { methylene }\end{array}$ & Optional & Optional & Unknown \\
\hline $\begin{array}{l}\text { Elmadahm et al. } \\
(2015) \text { [21] }\end{array}$ & $\mathrm{RCT}$ & Australia & 1088 & Unknown & Negative & No & ${ }^{99 \mathrm{~m}} \mathrm{Tc}$-sulfur colloid & Patent blue & Peritumoural & Peritumoural & 779/957 (81.4 \%) \\
\hline $\begin{array}{l}\text { O'Reilly et al. (2015) } \\
\text { [11] }\end{array}$ & $\mathrm{RCT}$ & Ireland & 667 & 48 & Negative & No & Unknown & Isosulfan & Intradermal & Subdermal & $667 / 667(100 \%)$ \\
\hline
\end{tabular}

Johnson et al.

Unfiltered ${ }^{99 m} \mathrm{Tc}$

[3]

(2013) [20]

[11]

FNR false-negative rate, LSG lymphoscintigraphy, NAC neoadjuvant chemotherapy, NPS non-randomized prospective study 
Table 2 Quality assessment of included studies by a revised 6-item scale

\begin{tabular}{|c|c|c|c|c|c|c|c|}
\hline Author (year) & $\begin{array}{l}\text { Description of } \\
\text { patients' } \\
\text { characteristics }\end{array}$ & $\begin{array}{l}\text { Reasons for } \\
\text { withdrawal }\end{array}$ & $\begin{array}{l}\text { Description of } \\
\text { measures of } \\
\text { outcomes }\end{array}$ & $\begin{array}{l}\text { Evaluation of } \\
\text { confounding factors }\end{array}$ & $\begin{array}{l}\text { Description of the SLN } \\
\text { technique }\end{array}$ & $\begin{array}{l}\text { Sample size } \\
\text { over } 300\end{array}$ & $\begin{array}{l}\text { Total } \\
\text { score }\end{array}$ \\
\hline $\begin{array}{l}\text { Bass et al. } \\
\text { (1999) [18] }\end{array}$ & 0 & 0 & 1 & 1 & 1 & 1 & 4 \\
\hline $\begin{array}{l}\text { Mariani et al. } \\
\text { (2000) [28] }\end{array}$ & 1 & 0 & 1 & 0 & 1 & 0 & 3 \\
\hline $\begin{array}{l}\text { Rahusen et al. } \\
\text { (2000) [30] }\end{array}$ & 1 & 0 & 1 & 0 & 1 & 0 & 3 \\
\hline $\begin{array}{l}\text { Derossis et al. } \\
\text { (2001) [12] }\end{array}$ & 1 & 0 & 1 & 1 & 1 & 1 & 5 \\
\hline $\begin{array}{l}\text { Bauer et al. } \\
\text { (2002) [19] }\end{array}$ & 1 & 0 & 1 & 1 & 1 & 1 & 5 \\
\hline $\begin{array}{l}\text { Pelosi et al. } \\
\text { (2003) [29] }\end{array}$ & 1 & 0 & 1 & 1 & 1 & 0 & 4 \\
\hline $\begin{array}{l}\text { Fleming et al. } \\
\text { (2003) [22] }\end{array}$ & 1 & 0 & 1 & 1 & 1 & 0 & 4 \\
\hline $\begin{array}{l}\text { Ahrendt et al. } \\
(2002) \text { [17] }\end{array}$ & 1 & 0 & 1 & 1 & 1 & 0 & 4 \\
\hline $\begin{array}{l}\text { Tsunoda et al. } \\
\text { (2002) [34] }\end{array}$ & 0 & 0 & 1 & 1 & 1 & 1 & 4 \\
\hline $\begin{array}{l}\text { Schirrmeister } \\
\text { et al. (2004) [32] }\end{array}$ & 1 & 0 & 1 & 0 & 0 & 1 & 3 \\
\hline $\begin{array}{l}\text { Lauridsen et al. } \\
\text { (2004) [24] }\end{array}$ & 1 & 1 & 1 & 1 & 1 & 0 & 5 \\
\hline $\begin{array}{l}\text { Mamounas et al. } \\
\text { (2005) [27] }\end{array}$ & 1 & 1 & 1 & 1 & 0 & 1 & 5 \\
\hline $\begin{array}{l}\text { Takei et al. } \\
\text { (2006) [33] }\end{array}$ & 1 & 0 & 1 & 1 & 1 & 1 & 5 \\
\hline $\begin{array}{l}\text { Argon et al. } \\
\text { (2006) [1] }\end{array}$ & 1 & 0 & 1 & 1 & 1 & 0 & 4 \\
\hline $\begin{array}{l}\text { Low et al. } \\
\text { (2006) [26] }\end{array}$ & 1 & 1 & 1 & 1 & 1 & 0 & 5 \\
\hline $\begin{array}{l}\text { Goyal et al. } \\
\text { (2006) [23] }\end{array}$ & 1 & 0 & 1 & 1 & 1 & 1 & 5 \\
\hline $\begin{array}{l}\text { Lelievre et al. } \\
\text { (2007) [25] }\end{array}$ & 1 & 0 & 1 & 1 & 1 & 0 & 4 \\
\hline $\begin{array}{l}\text { Rodier et al. } \\
\text { (2007) [31] }\end{array}$ & 1 & 1 & 1 & 1 & 1 & 1 & 6 \\
\hline $\begin{array}{l}\text { Kang et al. } \\
\text { (2010) [10] }\end{array}$ & 1 & 0 & 1 & 1 & 0 & 1 & 4 \\
\hline $\begin{array}{l}\text { Johnson et al. } \\
\text { (2011) [8] }\end{array}$ & 0 & 0 & 1 & 1 & 1 & 1 & 4 \\
\hline $\begin{array}{l}\text { Kuehn et al. } \\
\text { (2013) [3] }\end{array}$ & 1 & 1 & 1 & 1 & 0 & 1 & 5 \\
\hline $\begin{array}{l}\text { Elmadahm et al. } \\
\text { (2015) [21] }\end{array}$ & 1 & 0 & 1 & 1 & 1 & 1 & 5 \\
\hline $\begin{array}{l}\text { O'Reilly et al. } \\
\text { (2015) [11] }\end{array}$ & 1 & 1 & 1 & 1 & 1 & 1 & 6 \\
\hline $\begin{array}{l}\text { Boughey et al. } \\
\text { (2013) [20] }\end{array}$ & 1 & 1 & 1 & 1 & 1 & 1 & 6 \\
\hline
\end{tabular}

combined method $[3,20,27,32]$. Kuehn et al. reported the false-negative rate in two subgroups, and they were separately analyzed [3]. The combined use of radioisotope and blue dye did not significantly lower the false-negative rate when compared with radioisotope alone $(\mathrm{OR}=0.76,95 \%$ CI $0.44-1.29, P>0.05)$. No 


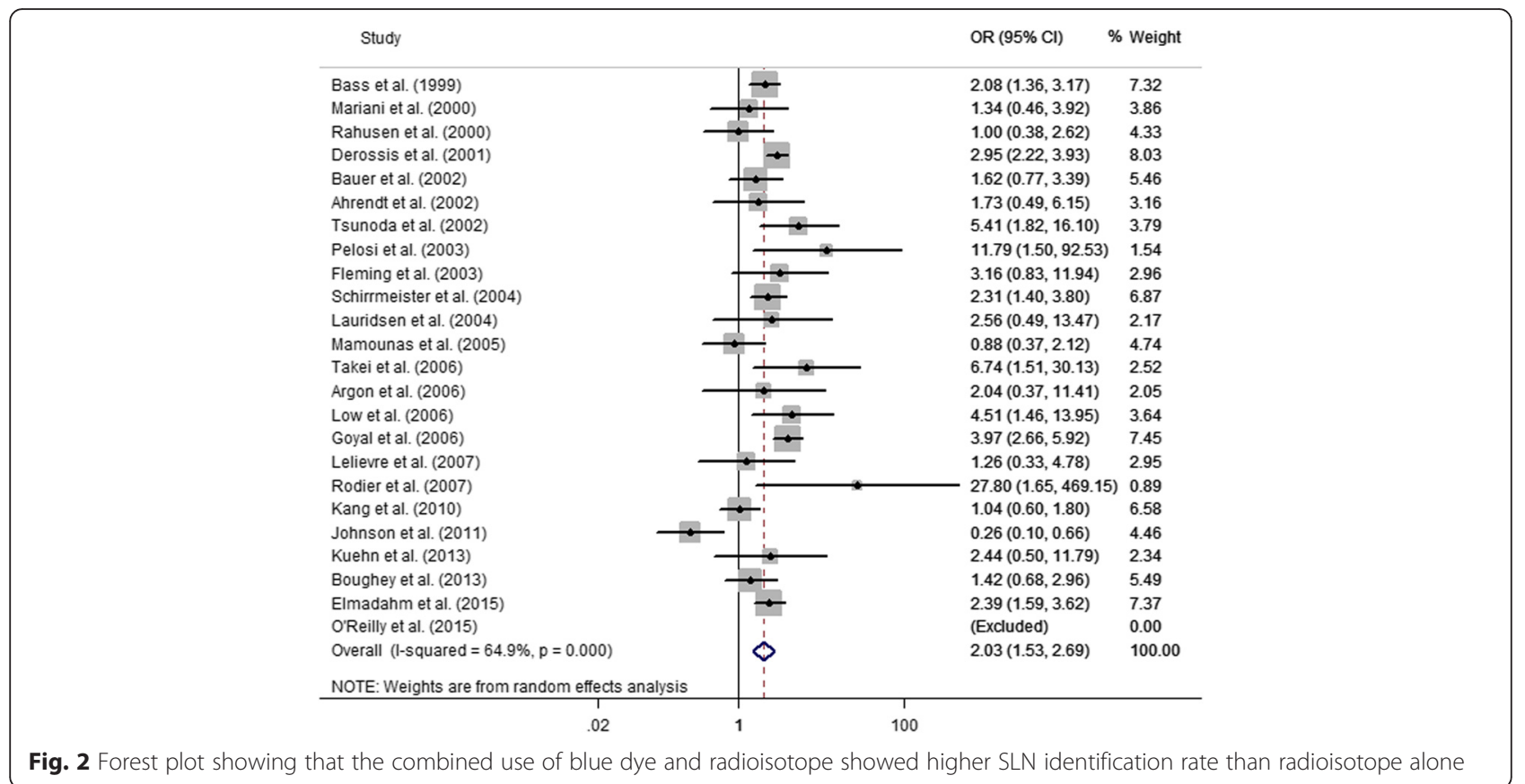

significant heterogeneity was detected $\left(\mathrm{I}^{2}=21.0 \%\right.$, $P>0.05$ ) (Fig. 5b).

\section{Adverse reactions}

Of the 24 publications, no study reported adverse episodes for the use of radioisotope. In contrast, 4 studies reported allergic reactions to blue dye $[1,10$, 11, 21]. Most patients experienced mild allergic reactions. However, Kang et al. reported 5 cases of serious allergic reactions presenting as hypotension among 2049 patients [10]. The pooled incidence of allergic reaction to blue dye was $0.6 \%$ (95\% CI $0.2-1.7 \%$ ),

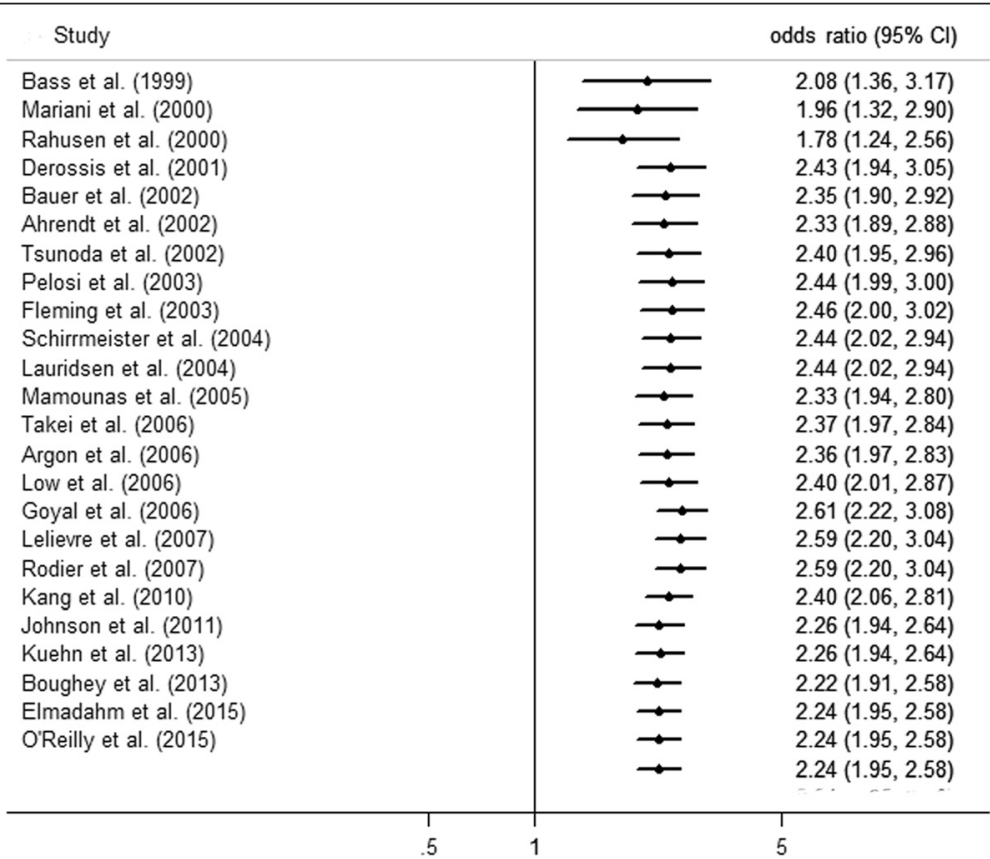

Fig. 3 Cumulative meta-analysis according to the publication year showing that the advantage of dual tracers remained stable over years 
Table 3 Subgroup analyses of studies on the sentinel lymph node identification

\begin{tabular}{|c|c|c|c|c|c|}
\hline Subgroups & No. of studies & OR & $95 \% \mathrm{Cl}$ & $P$ value & Heterogeneity $\left(I^{2}\right)$ \\
\hline \multicolumn{6}{|c|}{ Clinical node status } \\
\hline Negative & 14 & 2.56 & $1.88-3.49$ & $<0.05$ & 48.7 \\
\hline Positive & 1 & 1.42 & $0.68-2.96$ & $>0.05$ & - \\
\hline Mixed & 4 & 1.93 & $0.99-3.76$ & $>0.05$ & 59.5 \\
\hline \multicolumn{6}{|l|}{ NAC } \\
\hline Before NAC & 6 & 2.96 & $1.78-4.94$ & $<0.05$ & 15.6 \\
\hline After NAC & 3 & 1.53 & $0.94-2.47$ & $>0.05$ & 31.6 \\
\hline \multicolumn{6}{|c|}{ Proportion of patients with positive LSG } \\
\hline$\geq 90 \%$ & 7 & 1.41 & $0.83-2.39$ & $>0.05$ & 22.1 \\
\hline$<90 \%$ & 6 & 2.99 & $1.99-4.48$ & $<0.05$ & 42.8 \\
\hline \multicolumn{6}{|l|}{ Data source } \\
\hline $\mathrm{RCT}$ & 6 & 2.25 & $1.23-4.11$ & $<0.05$ & $74.7 \%$ \\
\hline NPS & 18 & 1.96 & $1.40-2.74$ & $<0.05$ & $62.5 \%$ \\
\hline \multicolumn{6}{|l|}{ Sample size } \\
\hline$>300$ & 15 & 2.01 & $1.42-2.84$ & $<0.05$ & $75.9 \%$ \\
\hline$<300$ & 9 & 2.01 & $1.28-3.15$ & $<0.05$ & $7.0 \%$ \\
\hline \multicolumn{6}{|l|}{ Location } \\
\hline USA & 8 & 1.33 & $0.82-2.16$ & $>0.05$ & $80.1 \%$ \\
\hline Europe & 11 & 2.48 & $1.60-3.84$ & $<0.05$ & $43.7 \%$ \\
\hline Asia & 5 & 2.93 & $2.05-4.19$ & $<0.05$ & $1.3 \%$ \\
\hline \multicolumn{6}{|c|}{ Injection site of blue dye } \\
\hline Superficial & 10 & 1.95 & $0.93-4.08$ & $>0.05$ & $70.6 \%$ \\
\hline Deep & 9 & 2.76 & $2.32-3.30$ & $<0.05$ & 0 \\
\hline \multicolumn{6}{|c|}{ Injection site of radioisotope } \\
\hline Superficial & 9 & 2.05 & $0.87-4.84$ & $>0.05$ & $76.0 \%$ \\
\hline Deep & 11 & 2.55 & $1.93-3.37$ & $<0.05$ & $31.2 \%$ \\
\hline
\end{tabular}

LSG lymphoscintigraphy, NAC neoadjuvant chemotherapy

with significant heterogeneity $\left(\mathrm{I}^{2}=72.5 \%, P<0.05\right)$ (Fig. 6).

\section{Discussion}

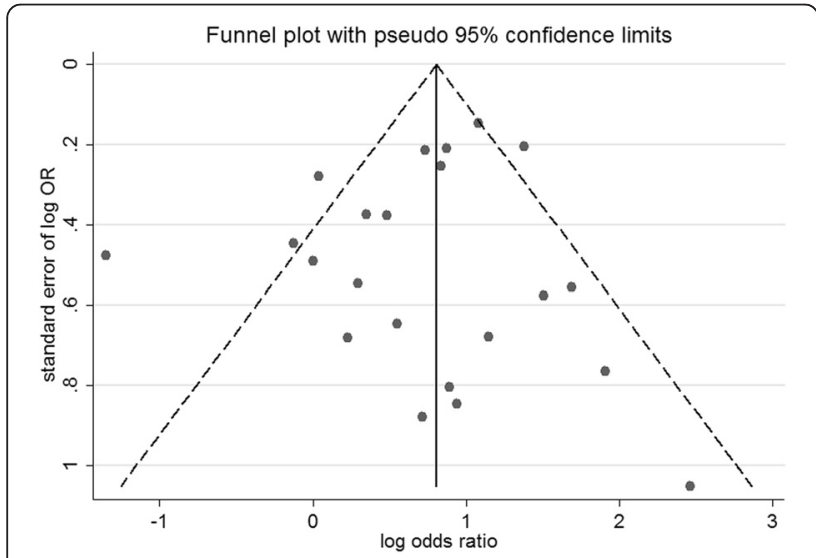

Fig. 4 Funnel plot showing that no evidence of publication bias was identified
This systematic review included 24 studies involving 15,462 participants. To our knowledge, it represented the largest and most comprehensive systematic review of prospective studies investigating the added value of blue dye in addition to radioisotope alone for tracing SLNs in breast cancer. It reflected the worldwide experience over 20 years. The overall pooled analysis showed that the combination of radioisotope and blue dye was superior to radioisotope alone for the successful identification of SLNs. The pooled false-negative rate was $7.5 \%$, which was similar to the pooled rate of $7.3 \%$ in a previous meta-analysis [13]. Notably, the combined use of blue dye and radioisotope failed to confer significant advantage in lowering the false-negative rate.

The recent RCT failed to demonstrate an advantage with the addition of blue dye to radioisotope alone in 
A

Study name

Bass et al. (1999)

Ahrendt et al. (2002)

Fleming et al. (2003)

Schirrmeister et al. (2004)

Lauridsen et al. (2004)

Mamounas et al. (2005)

Argon et al. (2006)

Low et al. (2006)

Goyal et al. (2006)

Lelievre et al. (2007)

Kuehn et al. (2013)

\section{Event rate and $95 \% \mathrm{Cl}$}

$\begin{array}{ccc}\begin{array}{c}\text { Event } \\ \text { rate }\end{array} & \begin{array}{c}\text { Lower } \\ \text { limit }\end{array} & \begin{array}{c}\text { Upper } \\ \text { limit }\end{array} \\ 0.008 & 0.001 & 0.057 \\ 0.037 & 0.009 & 0.136 \\ 0.067 & 0.025 & 0.165 \\ 0.083 & 0.053 & 0.128 \\ 0.015 & 0.002 & 0.100 \\ 0.107 & 0.066 & 0.170 \\ 0.045 & 0.003 & 0.448 \\ 0.029 & 0.004 & 0.181 \\ 0.067 & 0.043 & 0.103 \\ 0.066 & 0.036 & 0.118 \\ 0.224 & 0.180 & 0.276 \\ 0.066 & 0.038 & 0.111\end{array}$

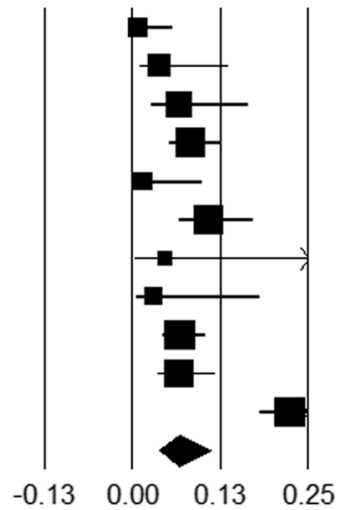

False-negative rate

B

\begin{tabular}{|c|c|c|}
\hline Study & OR $(95 \% \mathrm{Cl}) \quad \%$ & Weight \\
\hline Schirrmeister et al. (2004) & $1.10(0.30,4.02)$ & 14.71 \\
\hline Mamounas et al. (2005) & $1.96(0.23,16.89)$ & 5.86 \\
\hline Kuehn et al. Arm B (2013) & $1.48(0.54,4.08)$ & 21.89 \\
\hline Kuehn et al. Arm C (2013) & $0.49(0.19,1.27)$ & 24.08 \\
\hline Boughey et al. (2013) & $0.47(0.22,1.00)$ & 33.45 \\
\hline Overall (I-squared $=21.0 \%, p=0.281$ ) & $0.76(0.44,1.29)$ & 100.00 \\
\hline
\end{tabular}

Fig. 5 Forest plots showing the pooled false-negative rate, and the comparison between dual tracers and radioisotope alone in false-negative rate. a pooled false-negative rate $\mathbf{b}$ Forest plot of ORs showing that the combination of blue dye and radioisotope did not significantly decrease the false-negative rate when compared with radioisotope alone

patients before NAC with positive preoperative LSG [11]. LSG is a useful tool to establish abnormal lymphatic drainage patterns, and to detect extra-axillary nodes particularly internal mammary nodes [21, 24, 30]. It is an integral part of single radioisotope tracer during SLNB. Half of our included studies have performed

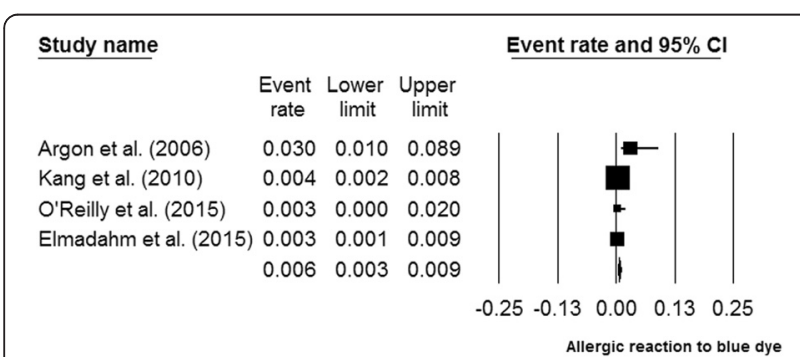

Fig. 6 Forest plot showing the pooled incidence of allergic reaction to blue dye preoperative LSG. When pooling results for studies enrolling over $90 \%$ patients with positive LSG, no advantage was demonstrated for using dual tracers. Meta-regression analysis revealed that preoperative LSG appeared to be the source of heterogeneity. These results were consistent with the recent trial that positive preoperative LSG may preclude the additional use of blue dye [11]. Several studies have suggested that the uptake of radioisotope was less favorable after NAC compared with primary surgery, which may decrease the detection rate of SLNs [3, 35, 36]. Nevertheless, we failed to show the advantage of dual tracers for patients after NAC. Additionally, the statistical significance was not shown in the subgroups of superficial injection of blue dye or radioisotope. For a long time, the optimal injection sites of mapping agents were controversial. A previous metaanalysis suggested that both superficial and deep injections of radioisotope and blue dye were effective for identifying 
SLNs, but failing to show statistical difference between the two methods [16]. Interestingly, in 3 studies comparing superficial injection with deep injection, the blue and hot concordance was higher in the superficial-injection group compared with the deep-injection group (90\% versus $87 \%, 95.5 \%$ versus $85.5 \%$, and $95.6 \%$ versus $91.5 \%$, respectively) [19, 22, 31]. Thus, we inferred that the high concordance of blue dye and radioisotope for superficial injection may weaken the additional value of blue dye.

The learning curve was associated with the successful rate of SLN identification. It was estimated that 23 patients were required by an individual surgeon to achieve a $90 \% \pm 4.5 \%$ success rate and 53 patients were required to achieve a $95 \% \pm 2.3 \%$ success rate [18]. The marginal benefit of blue dye was shown to be significant during the initial learning period, but declining with increased experience in using radioisotope alone $[10,12]$. Assuming that breast surgeons have gained more experience in mapping techniques during the recent years, cumulative and meta-regression analyses were conducted according to the publication year. However, no statistical significance was detected.

One major concern for blue dye was the potential risk of complications, which were infrequent but significant, including anaphylaxis, skin tattooing, and skin necrosis at the injection site [8]. Our results showed that the incidence of allergic reaction to blue dye was at a low level of $0.6 \%$. The allergic reactions to blue dye were categorized into grade 1 (urticaria or blue hives, pruritis, or a generalized rash), grade 2 (hypotension not requiring vasopressors), or grade 3 (hypotension requiring pressor support) [37]. Most allergic episodes in our included studies were of grade 1, and only Kang et al. reported the hypotension episodes of grade $2(5 / 2049)$ and grade 3 (2/2049) [10]. In addition, blue dye may be unavailable in some institutions due to nationwide shortage. The localization of SLNs was surgeon-dependent for mapping with blue dye [18]. Although the cost of methylene blue was low, the charge for lymphazurin reached as high as approximately $\$ 600$ in the USA [8]. Thus, the conversion from dual tracers to a single radioisotope injection would help facilitate the biopsy procedure, reduce complications, and diminished cost as well as resource utilization.

Several limitations of our study should be acknowledged. The number of included studies was small for the outcome of false-negative rate. Only a minority of the selected studies recorded the events of allergic reactions to blue dye in follow-up. Especially, only 1 RCT was primarily designed to compare dual tracers with radioisotope alone [11]. Most studies were non-randomized studies or post-hoc analyses of RCTs. The reason for choice of tracer was unknown for most studies. Thus, the selection bias may exist. It was difficult to match the age, race, distribution of clinical stages, relation to NAC, type of radiotracer, injection site of mapping agents, and surgeons' experience between the comparison groups. These confounding factors may affect the identification rate as well as the false-negative rate. Skip metastasis, intraoperative pathological technique, and lymphatic vessel obstruction have been suggested to the main reasons for false-negative results [38], which may overweigh the influence of mapping-agents choice.

\section{Conclusion}

In conclusion, compared with radioisotope alone, blue dye plus radioisotope showed a higher success rate of SLN identification. Nevertheless, the added value of blue dye appeared to be limited for patients with positive preoperative LSG, having received NAC, or undergoing superficial injection of tracing agents. Dual tracers were unhelpful for lowering the false-negative rate of SLNs. Considering the adverse reactions and inconvenience caused by blue dye injection, and the increased experience in using radioisotope, the advantage of dual tracers over radioisotope alone may be overestimated. Further well-designed randomized studies are required to recognize the sub-population in whom the dual tracers is especially required.

\section{Competing interests}

The authors declare that they have no competing interests.

\section{Authors' contributions}

PSH and FL conducted the study and drafted the manuscript. PSH and GHL searched the databases, and participated in the extraction and analysis of data. CG and TJC conceived the study, participated in its design and coordination and helped to draft the manuscript. All of the authors read and approved the final manuscript

\section{Acknowledgements}

None.

\section{Author details}

${ }^{1}$ The Second Department of General Surgery, Chongqing Three Gorges Central Hospital, 165 Xincheng Road, Chongqing 404000, China.

Department of Breast Surgery, Three Gorges Central Hospital, 165 Xincheng Road, Chongqing 404000, China. ${ }^{3}$ Department of Urinary Surgery, Three Gorges Central Hospital, 165 Xincheng Road, Chongqing 404000, China.

Received: 23 September 2015 Accepted: 7 February 2016

Published online: 16 February 2016

\section{References}

1. Argon AM, Duygun U, Acar E, Daglioz G, Yenjay L, Zekioglu O, et al. The use of periareolar intradermal Tc-99m tin colloid and peritumoral

intraparenchymal isosulfan blue dye injections for determination of the sentinel lymph node. Clin Nucl Med. 2006;31(12):795-800. doi:10.1097/01.rlu. 0000246855.80027.b7.

2. Lyman GH, Temin S, Edge SB, Newman LA, Turner RR, Weaver DL, et al. Sentinel lymph node biopsy for patients with early-stage breast cancer: American Society of Clinical Oncology clinical practice guideline update. J Clin Oncol. 2014;32(13):1365-83. doi:10.1200/JCO.2013.54.1177.

3. Kuehn T, Bauerfeind I, Fehm T, Fleige B, Hausschild M, Helms G, et al. Sentinel-lymph-node biopsy in patients with breast cancer before and after neoadjuvant chemotherapy (SENTINA): a prospective, multicentre cohort study. Lancet Oncol. 2013;14(7):609-18. doi:10.1016/S1470-2045(13)70166-9.

4. Giuliano AE, Kirgan DM, Guenther JM, Morton DL. Lymphatic mapping and sentinel lymphadenectomy for breast cancer. Ann Surg. 1994;220(3):391-8. discussion 8-401. 
5. Krag DN, Weaver DL, Alex JC, Fairbank JT. Surgical resection and radiolocalization of the sentinel lymph node in breast cancer using a gamma probe. Surg Oncol. 1993;2(6):335-9. discussion 40.

6. Albertini JJ, Lyman GH, Cox C, Yeatman T, Balducci L, Ku N, et al. Lymphatic mapping and sentinel node biopsy in the patient with breast cancer. JAMA. 1996:276(22):1818-22.

7. Lyman GH, Giuliano AE, Somerfield MR, Benson 3rd AB, Bodurka DC, Burstein $\mathrm{HJ}$, et al. American Society of Clinical Oncology guideline recommendations for sentinel lymph node biopsy in early-stage breast cancer. J Clin Oncol. 2005:23(30):7703-20. doi:10.1200/JCO.2005.08.001.

8. Johnson CB, Boneti C, Korourian S, Adkins L, Klimberg VS. Intraoperative injection of subareolar or dermal radioisotope results in predictable identification of sentinel lymph nodes in breast cancer. Ann Surg. 2011; 254(4):612-8. doi:10.1097/SLA.0b013e31823005bf.

9. Bines S, Kopkash K, Ali A, Fogg L, Wool N. The use of radioisotope combined with isosulfan Blue dye is not superior to radioisotope alone for the identification of sentinel lymph nodes in patients with breast cancer. Surgery. 2008;144(4):606-9. doi:10.1016/j.surg.2008.06.023. discussion 9-10.

10. Kang T, Yi M, Hunt KK, Mittendorf EA, Babiera GV, Kuerer H, et al. Does blue dye contribute to success of sentinel node mapping for breast cancer? Ann Surg Oncol. 2010;17 Suppl 3:280-5. doi:10.1245/s10434-010-1235-5.

11. O'Reilly EA, Prichard RS, Al Azawi D, Aucharaz N, Kelly G, Evoy D, et al. The value of isosulfan blue dye in addition to isotope scanning in the identification of the sentinel lymph node in breast cancer patients with a positive lymphoscintigraphy: a randomized controlled trial (ISRCTN98849733). Ann Surg. 2015. doi:10.1097/sla.0000000000001213.

12. Derossis AM, Fey J, Yeung $H$, Yeh SD, Heerdt AS, Petrek J, et al. A trend analysis of the relative value of blue dye and isotope localization in 2,000 consecutive cases of sentinel node biopsy for breast cancer. J Am Coll Surg. 2001;193(5):473-8

13. Kim T, Giuliano AE, Lyman GH. Lymphatic mapping and sentinel lymph node biopsy in early-stage breast carcinoma: a metaanalysis. Cancer. 2006; 106(1):4-16. doi:10.1002/cncr.21568.

14. Dupont WD, Plummer Jr WD. Power and sample size calculations. A review and computer program. Control Clin Trials. 1990;11(2):116-28.

15. DerSimonian R, Laird N. Meta-analysis in clinical trials. Control Clin Trials. 1986;7(3):177-88.

16. Ahmed M, Purushotham AD, Horgan K, Klaase JM, Douek M. Meta-analysis of superficial versus deep injection of radioactive tracer and blue dye for lymphatic mapping and detection of sentinel lymph nodes in breast cancer. Br J Surg. 2015;102(3):169-81. doi:10.1002/bjs.9673.

17. Ahrendt GM, Laud P, Tjoe J, Eastwood D, Walker AP, Otterson MF, et al. Does breast tumor location influence success of sentinel lymph node biopsy? J Am Coll Surg. 2002;194(3):278-84.

18. Bass SS, Cox CE, Ku NN, Berman C, Reintgen DS. The role of sentinel lymph node biopsy in breast cancer. J Am Coll Surg. 1999;189(2):183-94.

19. Bauer TW, Spitz FR, Callans LS, Alavi A, Mick R, Weinstein SP, et al. Subareolar and peritumoral injection identify similar sentinel nodes for breast cancer. Ann Surg Oncol. 2002;9(2):169-76.

20. Boughey JC, Suman VJ, Mittendorf EA, Ahrendt GM, Wilke LG, Taback B, et al. Sentinel lymph node surgery after neoadjuvant chemotherapy in patients with node-positive breast cancer: the ACOSOG Z1071 (Alliance) clinical trial. JAMA. 2013;310(14):1455-61. doi:10.1001/jama.2013.278932.

21. Elmadahm AA, Gill PG, Bochner M, Gebski VJ, Zannino D, Wetzig N, et al. Identification of the sentinel lymph node in the SNAC-1 trial. ANZ J Surg. 2015;85(1-2):58-63. doi:10.1111/ans.12527.

22. Fleming FJ, Hill AD, Kavanagh D, Quinn C, O'Doherty A, Collins CD, et al. Intradermal radioisotope injection optimises sentinel lymph node identification in breast cancer. Eur J Surg Oncol. 2003;29(10):835-8.

23. Goyal A, Newcombe RG, Chhabra A, Mansel RE. Factors affecting failed localisation and false-negative rates of sentinel node biopsy in breast cancer-results of the ALMANAC validation phase. Breast Cancer Res Treat. 2006;99(2):203-8. doi:10.1007/s10549-006-9192-1.

24. Lauridsen MC, Garne JP, Sorensen FB, Melsen F, Lernevall A, Christiansen P. Sentinel lymph node biopsy in breast cancer-experience with the combined use of dye and radioactive tracer at Aarhus University Hospital. Acta Oncol. 2004;43(1):20-6.

25. Lelievre L, Houvenaeghel G, Buttarelli M, Brenot-Rossi I, Huiart L, Tallet A, et al. Value of the sentinel lymph node procedure in patients with large size breast cancer. Ann Surg Oncol. 2007:14(2):621-6. doi:10.1245/s10434-006-9232-4.
26. Low KS, Littlejohn DR. Optimal number of sentinel nodes after intradermal injection isotope and blue dye. ANZ J Surg. 2006;76(6):472-5. doi:10.1111/j. 1445-2197.2006.03752.x.

27. Mamounas EP, Brown A, Anderson S, Smith R, Julian T, Miller B, et al. Sentinel node biopsy after neoadjuvant chemotherapy in breast cancer: results from National Surgical Adjuvant Breast and Bowel Project Protocol B-27. J Clin Oncol. 2005;23(12):2694-702. doi:10.1200/jco.2005.05.188.

28. Mariani G, Villa G, Gipponi M, Bianchi P, Buffoni F, Agnese G, et al. Mapping sentinel lymph node in breast cancer by combined lymphoscintigraphy, blue-dye, and intraoperative gamma-probe. Cancer Biother Radiopharm. 2000;15(3):245-52. doi:10.1089/108497800414338.

29. Pelosi E, Baiocco C, Ala A, Gay E, Bello M, Varetto T, et al. Lymphatic mapping in early stage breast cancer: comparison between periareolar and subdermal injection. Nucl Med Commun. 2003;24(5):519-23. doi:10.1097/01. mnh.0000071245.54690.7a.

30. Rahusen FD, Pijpers R, Van Diest PJ, Bleichrodt RP, Torrenga H, Meijer S. The implementation of the sentinel node biopsy as a routine procedure for patients with breast cancer. Surgery. 2000;128(1):6-12. doi:10.1067/msy.2000.107229.

31. Rodier JF, Velten M, Wilt M, Martel P, Ferron G, Vaini-Elies V, et al. Prospective multicentric randomized study comparing periareolar and peritumoral injection of radiotracer and blue dye for the detection of sentinel lymph node in breast sparing procedures: FRANSENODE trial. J Clin Oncol. 2007;25(24):3664-9. doi:10.1200/jco.2006.08.4228.

32. Schirrmeister H, Kotzerke J, Vogl F, Buck A, Czech N, Koretz K, et al. Prospective evaluation of factors influencing success rates of sentinel node biopsy in 814 breast cancer patients. Cancer Biother Radiopharm. 2004;19(6): 784-90. doi:10.1089/cbr.2004.19.784.

33. Takei H, Suemasu K, Kurosumi M, Horii Y, Ninomiya J, Kamimura M, et al. Added value of the presence of blue nodes or hot nodes in sentinel lymph node biopsy of breast cancer. Breast Cancer. 2006;13(2):179-85.

34. Tsunoda N, Iwata H, Sarumaru S, Mizutani M, Iwase T, Miura S. Combination of subareolar blue dye and peritumoral RI for sentinel lymph node biopsy. Breast Cancer. 2002:9(4):323-8.

35. Classe JM, Bordes V, Campion L, Mignotte H, Dravet F, Leveque J, et al. Sentinel lymph node biopsy after neoadjuvant chemotherapy for advanced breast cancer: results of Ganglion Sentinelle et Chimiotherapie Neoadjuvante, a French prospective multicentric study. J Clin Oncol. 2009; 27(5):726-32. doi:10.1200/JCO.2008.18.3228.

36. Hunt KK, Yi M, Mittendorf EA, Guerrero C, Babiera GV, Bedrosian I, et al. Sentinel lymph node surgery after neoadjuvant chemotherapy is accurate and reduces the need for axillary dissection in breast cancer patients. Ann Surg. 2009;250(4):558-66. doi:10.1097/SLA.0b013e3181b8fd5e.

37. Montgomery LL, Thorne AC, Van Zee KJ, Fey J, Heerdt AS, Gemignani M, et al. Isosulfan blue dye reactions during sentinel lymph node mapping for breast cancer. Anesth Analg. 2002;95(2):385-8. table of contents.

38. Nakashima K, Kurebayashi J, Sonoo H, Tanaka K, Ikeda M, Shiiki S, et al. Preoperative dynamic lymphoscintigraphy predicts sentinel lymph node metastasis in patients with early breast cancer. Breast Cancer. 2010;17(1):17-21. doi:10.1007/s12282-009-0123-y.

\section{Submit your next manuscript to BioMed Central and we will help you at every step:}

- We accept pre-submission inquiries

- Our selector tool helps you to find the most relevant journal

- We provide round the clock customer support

- Convenient online submission

- Thorough peer review

- Inclusion in PubMed and all major indexing services

- Maximum visibility for your research

Submit your manuscript at www.biomedcentral.com/submit 